\title{
TU/e EmonOWEN

\section{Determining porosity profiles during filtration and expression of sewage sludge by NMR imaging}

\section{Citation for published version (APA):}

Heij, la, E. J., Kopinga, K., Pel, L., \& Kerkhof, P. J. A. M. (1996). Determining porosity profiles during filtration and expression of sewage sludge by NMR imaging. AIChE Journal, 42(4), 953-959.

https://doi.org/10.1002/aic.690420408

DOI:

10.1002/aic.690420408

Document status and date:

Published: 01/01/1996

\section{Document Version:}

Publisher's PDF, also known as Version of Record (includes final page, issue and volume numbers)

\section{Please check the document version of this publication:}

- A submitted manuscript is the version of the article upon submission and before peer-review. There can be important differences between the submitted version and the official published version of record. People interested in the research are advised to contact the author for the final version of the publication, or visit the $\mathrm{DOI}$ to the publisher's website.

- The final author version and the galley proof are versions of the publication after peer review.

- The final published version features the final layout of the paper including the volume, issue and page numbers.

Link to publication

\section{General rights}

Copyright and moral rights for the publications made accessible in the public portal are retained by the authors and/or other copyright owners and it is a condition of accessing publications that users recognise and abide by the legal requirements associated with these rights.

- Users may download and print one copy of any publication from the public portal for the purpose of private study or research.

- You may not further distribute the material or use it for any profit-making activity or commercial gain

- You may freely distribute the URL identifying the publication in the public portal.

If the publication is distributed under the terms of Article 25fa of the Dutch Copyright Act, indicated by the "Taverne" license above, please follow below link for the End User Agreement:

www.tue.nl/taverne

Take down policy

If you believe that this document breaches copyright please contact us at:

openaccess@tue.nl

providing details and we will investigate your claim. 


\title{
Determining Porosity Profiles During Filtration and Expression of Sewage Sludge by NMR Imaging
}

\author{
E. J. La Heij and P. J. A. M. Kerkhof \\ Dept. of Chemical Engineering \\ K. Kopinga \\ Dept. of Physics \\ L. Pel \\ Dept. of Architecture and Building Technology \\ Eindhoven University of Technology, 5600 MB, Eindhoven, The Netherlands
}

\begin{abstract}
The formation of a filter cake during mechanical dewatering (filtration and expression) of sludge is studied using one-dimensional nuclear magnetic resonance (NMR) imaging of porosity profiles. The experiments clearly showed the formation of a porosity gradient during filtration and the disappearing of this gradient during expression, forming a cake with a uniform porosity. This technique makes it possible to investigate the material behavior of the porous medium, which is needed before any model calculations can be performed. The results indicate that NMR imaging is useful in nondestructive monitoring of the filtration and expression process of sludge.
\end{abstract}

\section{Introduction}

Modeling the liquid transport during filtration and expression of sludge, or any other liquid-solid mixture, is of great importance for the development of operation rules for mechanical dewatering equipment. Often overall dewatering parameters, like filtrate volume, average porosity, or cake thicknesses are compared to model calculations (Willis et al., 1983; Tiller and Yeh, 1987; Stamatakis and Tien, 1991; La Heij et al., 1994). A more fundamental approach to the test of model calculations is to measure the internal structure of filter cakes, i.e., porosity profiles.

Dewatering of compressible porous media can be distinguished into two phases, filtration and expression. In Figure 1 the mechanisms of filtration and expression are drawn for an experiment in a laboratory filter cell with axial liquid flow. After a piston is placed on top of the slurry, a cake builds up from the filter medium ( $d L / d t>0, L$ is cake thickness). The filtrate flows through the filter medium and the ratio of the pressure drop across the medium to the pressure drop across the cake decreases. As the cake builds up, the hydraulic pressure at the top of the cake and in the slurry remains constant (equal to the constant applied pressure on the piston). Going

Correspondence concerning this article should be addressed to P. J. A. M. Kerkhof from the slurry $\left(\epsilon_{s l}\right)$ to the cake $\left(\epsilon_{0}\right)$, there is a discontinuity in porosity. In the cake the particles have contact with each other, while in the slurry the particles float in the liquid without having contact. In the filter cake a porosity gradient is formed. This is caused by the decrease of the hydraulic pressure and the increase of the compressive pressure, which reflects the stress on the particles (and thus the local porosity in the filter cake). When the slurry has disappeared (the piston has reached the upper cake boundary), the expression phase starts and the cake is compressed $(d L / d t<0)$. During expression the piston presses on top of the filter cake, which results in a cake compression and a decrease of the cake thickness $L$. Due to this compression, the porosity gradient slowly disappears and finally a uniform equilibrium porosity $\left(\epsilon_{\infty}\right)$ results. Obviously, the solid matrix changes continuously during these phases. The applied filtration-expression pressure only affects the equilibrium porosity $\left(\epsilon_{\infty}\right)$ and has only a small influence on the dewatering rate if the filter cake is highly compressible (La Heij, 1994). In Figure 2 a theoretical representation of the development of porosity profiles is shown. The shape of the profile at the end of the filtration phase is determined by the compressibility of the material. Highly compressible materials will have a steep profile near 




Figure 1. Mechanism of filtration and expression (Tiller and Yeh, 1987).

$L$ is the cake thickness; $p$, is the hydraulic pressure.

the filter medium, i.e., a relatively dry cake near the filter medium.

Of course, porosity profiles can be calculated by models presented in the literature. Generally, however, these models are only hypothetical, since the resulting profiles never could be checked experimentally in a reliable way. By measuring the porosity profiles, the constitutive equations and the concept of the well-known compression-permeability cell (used to simulate the filtration and expression process) can be checked or, alternatively, the constitutive equations can be determined. Also, the boundary conditions and the basic assumptions of a model can be checked.

In most cases porosity profiles have been investigated using electrical resistance meters (Rietema, 1953; Shirato and Aragaki, 1972; Wakeman, 1981; Chase and Willis, 1991). During the measurements iron pins are positioned in the wall of the filter cell, which can disturb the liquid flow and cake

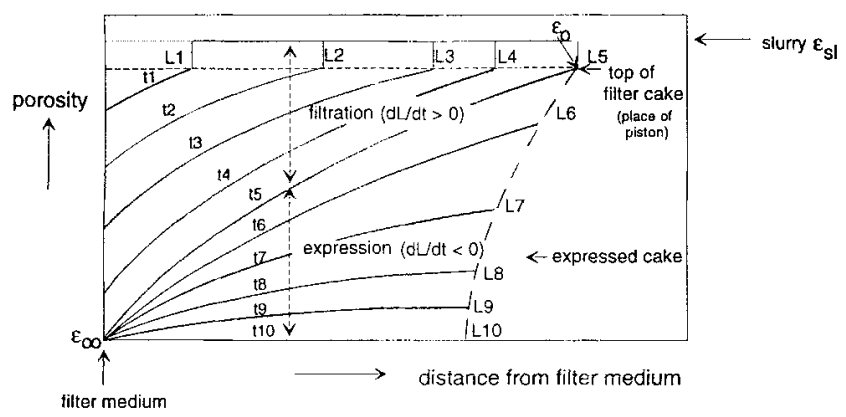

Figure 2. Theoretical diagram showing porosity profiles as a function of time during dewatering, starting from a slurry.

Two stages can be distinguished: (1) filtration, a filter cake is formed $(d L / d t>0)$ under the influence of slurry supply; and (2) expression, the slurry has been disappeared and the cake is compressed $(d L / d t<0) . \epsilon_{0}=$ porosity at top of the filter cake, which remains constant during the filtration phase; $\epsilon_{\infty}=$ final equilibrium porosity. Note that a discontinuity in porosity is present between the slurry and the filter cake. Labeled curves refer to profiles and cake thicknesses $\left(L_{i}\right)$ occurring at different times $\left(t_{i}\right)$. formation. Some investigators studied porosity profiles in filter cakes with X-rays (Bierck et al., 1988; Tiller et al., 1990) or nuclear magnetic resonance (NMR) techniques (Horsfield et al., 1989). Since these techniques are nondestructive, the cake structure is not influenced in any way, which is an advantage. An additional advantage of NMR is that it selectively probes the hydrogen nuclei. Moreover, with NMR a distinction can also be made between free and physically/chemically bound water. In the present study the porosity profiles in a sludge filter cake were measured by NMR imaging. The profiles are measured during the filtration as well as the expression phase, which, to our knowledge, has not been reported before.

\section{Theoretical Aspects}

To model the filtration and expression behavior of sewage sludge, attention must be focused on liquid flow through compressible porous media. Sludge filter cakes are highly compressible, i.e., the porosity of the cake changes significantly with pressure.

The mathematical formulation of mass transport in filter cakes at a macroscopic level is usually based on parabolic differential equations. If the gravity is neglected, the change of the porosity $\epsilon$ in time with respect to the material coordinate $\omega$ in a filter cake can be described by a nonlinear parabolic differential equation

$$
\left(\frac{\partial \epsilon}{\partial t}\right)_{\omega}=\frac{\partial}{\partial \omega}\left[-\frac{K}{\eta}(1-\epsilon) \frac{d p_{s}}{d \epsilon}\left(\frac{\partial \epsilon}{\partial \omega}\right)_{t}\right]
$$

In this equation $K$ is the permeability of the cake, $\eta$ the liquid viscosity, and $p_{s}$ the so-called compressive pressure ( $\mathrm{La}$ Heij, 1994). Equation 1 is derived in the Appendix. The permeability determines the dewatering rate. First, initial and boundary conditions have to be known before Eq. 1 can be solved. The initial condition for the filtration phase is that the porosity of the slurry is constant over the total height. During constant pressure filtration, the cake structure at the 
top of the filter cake remains unchanged. The hydraulic pressure $\left(p_{l}\right)$ equals the applied pressure. Therefore, the porosity at the top of the filter cake is equal to the unstressed porosity

$$
\epsilon=\epsilon_{0} \quad x=L(t) \quad t>0
$$

which can be seen from the stress balance (Tiller and Yeh, 1987)

$$
p_{l}+p_{s}=p
$$

If $p_{l}=p(=$ applied pressure $)$ then $p_{s}$ equals zero and the porosity equals the unstressed porosity (see also Eq. 9). At the filter medium, the solids velocity equals zero and the liquid flows through the filter medium, which can be considered as an incompressible porous medium. Therefore

$$
q_{l m}=\frac{K_{m}}{\eta} \frac{\Delta p_{l m}}{\Delta x_{m}}=\frac{\Delta p_{l m}}{R_{m} \eta} \quad x=0 \quad t>0
$$

where $K_{m}$ is the permeability of the filter medium, $\Delta x_{m}$ the thickness of the filter medium, $R_{m}$ the resistance of the filter medium, and $\Delta p_{l m}$ the hydraulic pressure drop over the filter medium. The initial condition for the expression phase will be determined by the porosity profile at the end of the filtration phase. Since the velocities of both the liquid and the solids are the same at the top of the cake, the Darcy equation

$$
\frac{\partial p_{l}}{\partial x}=-\frac{\partial p_{s}}{\partial x}=\frac{\eta}{K} \epsilon\left(v_{l}-v_{s}\right)
$$

and thus at the top of the filter cake the derivative of the porosity to the distance equals zero (assuming that $\epsilon$ is a unique function of $p_{s}$, see also Eq. 9)

$$
\frac{\partial \epsilon}{\partial x}=0 \quad x=L(t) \quad t>0
$$

Commonly, it is assumed that the porosity near the filter medium remains constant during constant pressure expression (if the filter medium resistance is neglected)

$$
\epsilon=\epsilon\left(p_{s, x=0}\right) \quad x=0 \quad t>0
$$

Secondly, constitutive equations have to be known which provide the relation between porosity, permeability, and compressive pressure. These relations are often determined using a compression-permeability cell (Shirato et al., 1968) or by fitting methods (Stamatakis and Tien, 1991). The dependence of the permeability and porosity on the compressive pressure is reflected by the so-called compressibility coefficients $\delta$ and $\lambda$, respectively (Tiller et al., 1980)

$$
\begin{aligned}
& K_{\infty}=K_{0}\left(1+\frac{p_{s}}{p_{a}}\right)^{-\delta} \\
& \epsilon_{\infty}=\epsilon_{0}\left(1+\frac{p_{s}}{p_{a}}\right)^{-\lambda}
\end{aligned}
$$

In these equations $K_{0}$ and $\epsilon_{0}$ are the permeability and porosity at $p_{s}=0$, respectively, and $p_{a}$ is a reference pressure. The index $\infty$ indicates that these values are determined in an equilibrium situation. The calculated shape of the porosity profiles is determined by $\delta$ and $\lambda$. By applying the constitutive Eqs. 8 and 9, Eq. 1 can be solved and the porosity profiles as a function of time can be calculated.

In an NMR experiment, the amplitude of the spin-echo signal, assuming a single exponential relaxation, and $T_{1} \gg T_{2}$, is given by

$$
S \propto \rho\left[1-\exp \left(\frac{-T_{R}}{T_{1}}\right)\right] \exp \left(\frac{-T_{E}}{T_{2}}\right)
$$

In this equation $\rho$ is the proton density, $T_{1}$ the spin-lattice or longitudinal relaxation time, $T_{2}$ the spin-spin or transverse relaxation time, $T_{R}$ the repetition time of the spin-echo experiment, and $T_{E}$ the so-called echo time (Hayashi et al., 1988). This relation holds for a homogeneous medium, for instance, a quartz tube filled with water. In sludges, however, the situation is more complicated. For water at the surface of a particle, $T_{2}$ will be decreased by surface relaxation with respect to that of free water. In the sludge and the cake, a broad distribution of particle and pore sizes is present. This will give rise to a distribution of transverse relaxation times, which depends on the porosity. For small values of the echo time $T_{E}$, the water at the particle surfaces or in the pores (small $T_{2}$ ), as well as the free water (larger $T_{2}$ ), will contribute to the spin-echo signal. At large values of $T_{E}$ on the other hand, the contribution of free water dominates. Since our objective is to determine the porosity profiles quantitatively, it is necessary to calibrate the NMR signal. We will return to this point in the next section.

\section{Materials and Methods}

Sludge samples were taken from the wastewater treatment plant in Eindhoven, The Netherlands. The sludge was a mixture of primary (nonbiochemically treated) and secondary (biochemically treated) sludge. The sludges were mixed with iron chloride and lime. Iron chloride and lime are flocculants and form together with the sludge particles aggregates in order to increase the permeability. First, the iron chloride is added to the sludge ( $7 \mathrm{wt} . \%, 100 \mathrm{~mL}$ ) and is mixed at 1,000 rpm for $15 \mathrm{~s}$. Thereafter, lime (weight percentage ranging from 20 to 50 ) is added and is mixed at $500 \mathrm{rpm}$ for $60 \mathrm{~s}$.

The filtration-expression experiments were performed using an NMR scanning apparatus of which an extensive description is given by Kopinga and Pel (1994). In this apparatus a constant magnetic field of $0.7 T$ (corresponding to NMR frequencies of about $30 \mathrm{MHz}$ ) is generated by a conventional iron-cored electromagnet. The probe head consists of a small filtration-expression cell with an inner diameter of $21 \mathrm{~mm}$. This cell is mounted inside a cylindrical coil with an ID of 35 $\mathrm{mm}$, made of seven turns of $\mathrm{Cu}$ wire. This coil forms part of a tuned LC circuit and is placed inside a shielded box.

To obtain a spin-echo signal from which quantitative information can be extracted, the LC circuit is carefully matched to the characteristic impedance of our equipment (50 $\Omega$ ). Moreaver, changes in the dielectric losses or detuning of the circuit due to variations of the moisture content of the sam- 
ple are minimized by a cylindrical Faraday shield, which is placed between the coil and the sample. Apart from this, the quality factor of the $\mathrm{LC}$ circuit has been reduced to $Q \approx 40$ by adding a small series resistor.

The NMR spin-echo signal is excited by straightforward Hahn pulse sequences. The radio frequency (RF) carrier signal is switched on for $18 \mu \mathrm{s}$ and $36 \mu \mathrm{s}$, corresponding to a 90 and 180 degree flipping angle of the nuclear spins, respectively. The repetition time $T_{R}$ was chosen at least 4 times as large as the spin-lattice relaxation time $T_{1}$, to eliminate the effect of $T_{1}$ on the magnitude of the spin-echo signal (see Eq. 10). To perform 1-D scans of the material present in the filtration-expression cell, magnetic field gradients of $0.3 \mathrm{~T} / \mathrm{m}$ are generated by a set of home built Anderson coils, yielding a $1-D$ resolution better than $1 \mathrm{~mm}$. At a certain carrier frequency $f_{\mathrm{RF}}$, the nuclear moments in a slice with a thickness of about $3 \mathrm{~mm}$ are excited. The resulting spin-echo signal is digitized and Fourier transformed, yielding an intensity profile over the thickness of that slice. By changing the carrier frequency, slices can be selected over a range of $25 \mathrm{~mm}$. By combining the profiles of a sufficient number of adjacent slices, the intensity profile over the entire sample can be obtained. This intensity profile is corrected for geometrical distortions such as those arising from the finite length of the coil of the LC circuit, with the help of a reference profile, measured using a quartz tube filled with water.

To relate the intensity profile to a porosity profile, a calibration of the equipment has to be performed. To this end various sludges and cakes with uniform porosities were prepared. The porosity of these calibration samples was determined gravimetrically; the resulting data were corrected for the contribution of bound water by means of differential thermal analysis. The NMR spin-echo signal of these samples was measured for a number of different values of $T_{E}$. The results are plotted in Figure 3. For each sample, the data can be represented by more or less straight lines. The slope of such a line yields the (apparent) relaxation rate $1 / T_{2}$ of that sample. The figure clearly reveals that the relaxation time decreases with decreasing porosity. This can be explained by the fact that as the porosity decreases, the fraction of free water (with a relatively large $T_{2}$ ) decreases more rapidly than the total water content.

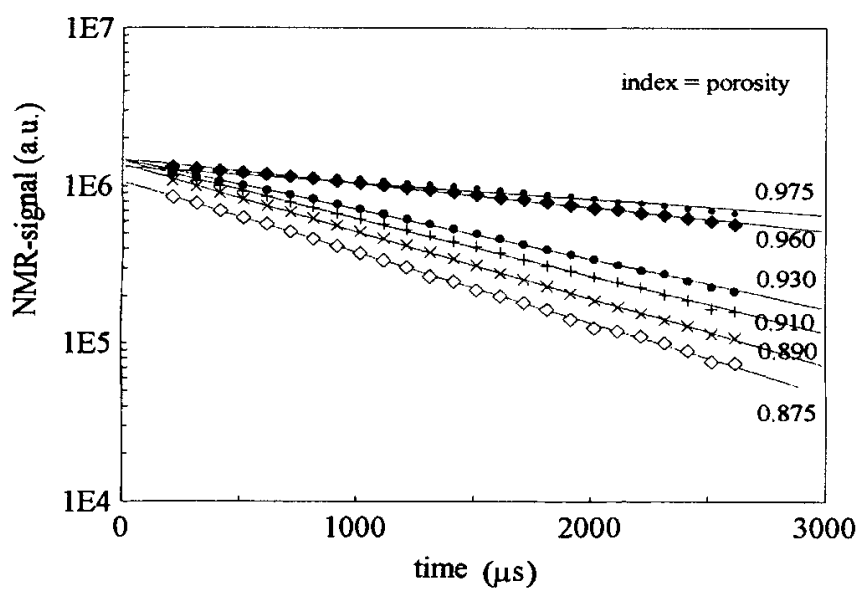

Figure 3. Measured NMR signal plotted against the corresponding spin-echo time $T_{E}$ for a fixed repetition time $T_{R}$ of $\mathbf{4 5 0}$ ms.

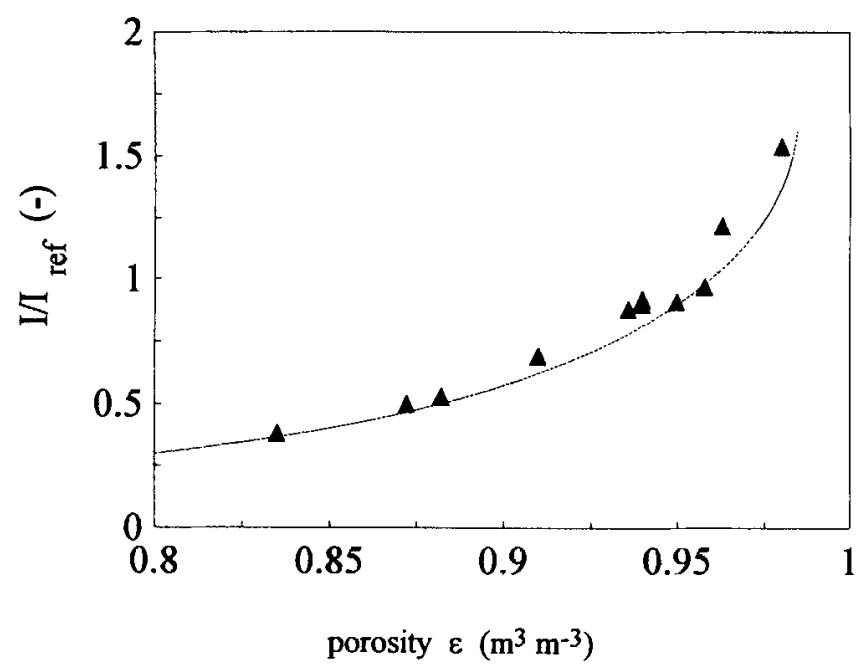

Figure 4. Calibration of the NMR signal.

The intensity observed for filter cakes with a uniform porosity, divided by the intensity of a reference sample, is plotted against the corresponding porosity.

The NMR signal is largest at small values of $T_{E}$, but in this region it varies only slightly with the porosity. At large values of $T_{E}$, on the other hand, the signal and hence the signal to noise ratio decreases, but the porosity contrast increases. Since the samples in our experiments cover only a limited range of porosities (variation of less than $15 \%$ ), the setting $T_{E}=1.8 \mathrm{~ms}$ was chosen as a (rather arbitrary) compromise between signal magnitude and contrast. This setting was fixed during all further calibrations and the actual experiments.

The calibration for $T_{E}=1.8 \mathrm{~ms}$ is shown in Figure 4. The experimental data (solid triangles) have an uncertainty of 0.005 and 0.015 along the horizontal and vertical axis, respectively. The dashed curve is a least-squares fit to the data and is used as a calibration. It is obvious that the NMR signal increases more rapidly than the porosity. This is caused by the facts that at $T_{E}=1.8 \mathrm{~ms}$ the signal arising from a certain amount of free water is larger than the signal arising from the same amount of water in pores or at particle surfaces, and that the fraction of free water increases at higher porosities. As such, this nonlinear relation is directly connected to the change of the apparent $T_{2}$ value with porosity presented in Figure 3.

With respect to the accuracy of the calibration, one should note that it does not permit an NMR measurement of the porosity with an absolute accuracy better than $1 \%$, but substantially smaller changes in the porosity can be detected unambiguously. Measurements in which $T_{R}$ was varied revealed that for sludge flocculated with iron chloride/lime $T_{1}$ was about $70 \mathrm{~ms}$, which is substantially smaller than the longitudinal relaxation time in pure water, and should be attributed to the presence of dissolved iron. From an experimental point of view, a small value of $T_{1}$ is favorable, since the repetition time $T_{R}$ can be relatively short and the porosity profiles can be measured quickly.

\section{Results and Discussion}

In Figure 5 an example of the experimentally determined porosity profiles is given for flocculated sludge ( $7 \mathrm{wt}$. \% iron 


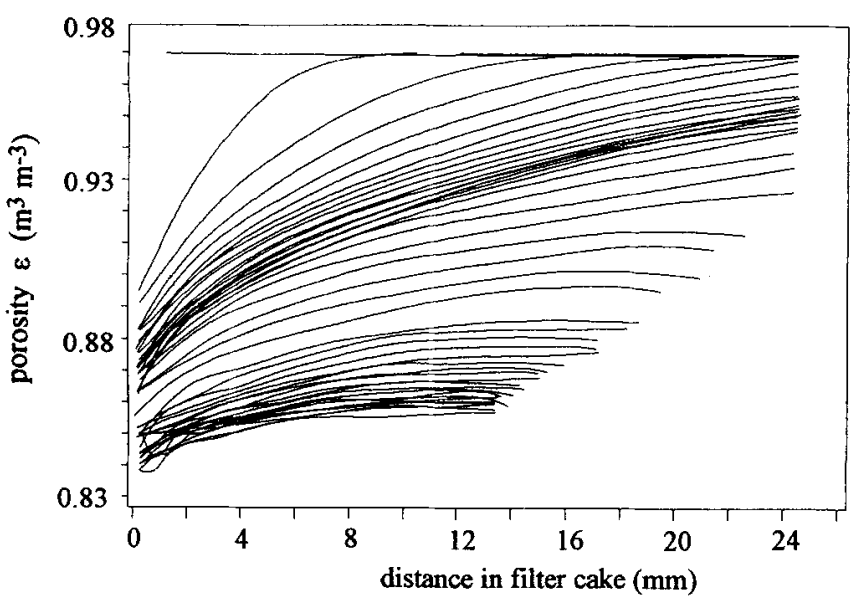

Figure 5. Porosity profiles in the filter cake as determined for sludge flocculated with $7 \mathrm{wt}$. \% iron chloride and $20 \mathrm{wt}$. \% lime on dry solids basis.

The time between the subsequent profiles is $160 \mathrm{~s}$. Total time of experiment is $180 \mathrm{~min}$. Boundary condition (applied pressure) $p=100 \mathrm{kPa} ; \eta=1.10^{3} \mathrm{~Pa} \cdot \mathbf{s} ; \boldsymbol{\epsilon}_{s l}=0.97$ (with flocculant); $\epsilon_{s l}=0.98$ (without flocculant).

chloride and $20 \mathrm{wt}$. \% lime on dry solids basis). The distance in the filter cake is measured from the filter medium. The time between the subsequent profiles is $160 \mathrm{~s}$; a lower limit to this time is set by the desired signal-to-noise ratio. The total duration of this experiment was two-and-a-half hours. Comparing Figures 2 and 5 clearly demonstrates that it is possible to measure porosity profiles in a sludge cake by use of NMR imaging. At the beginning of the experiment, a uniform porosity is present; the filter cell is completely filled with the sludge slurry. Then quite rapidly a certain porosity gradient develops, which slowly disappears during the expression phase, resulting finally in an almost uniform porosity. This is roughly in agreement with the theoretical assumptions, illustrated in Figure 1. In Figure 6 calculated porosity profiles for the expression phase are given. The time between the subsequent profiles is again $160 \mathrm{~s}$. The profiles do not end at $0.97\left(\epsilon_{0}\right)$ in this figure, because the initial cake had a thickness of $\pm 50 \mathrm{~mm}$ at the start of the expression phase, which is outside the figure. The constitutive parameters (determined by means of the compression-permeability cell) used in these calculations are: $K_{0}=3.0 \times 10^{-13} ; \epsilon_{0}=0.97 ; \delta=1.8$; $\lambda=0.15$. (These values were determined by plotting the compressive pressure vs. the measured porosity and permeability on a $\log -\log$ plot. The slopes will give the values of $\lambda$ and $\delta$, and the axis cut-off the values of $K_{0}$ and $\epsilon_{0}$. The exact value of these last two parameters is, however, difficult to determine, because a different value of $p_{a}$ (see Eqs. 8 and 9) gives another axis cutoff. The values of these parameters can be estimated from simulations and experiments, i.e., filtrate volume vs. time.) Since it is the purpose of the study reported here to observe whether or not qualitative differences occur between theory and measurement, rather than to minimize the quantitative deviation between measured and calculated profiles by adjusting the various parameter values, we preferred to plot the calculated and measured profiles in different figures. A comparison of the measured and calculated

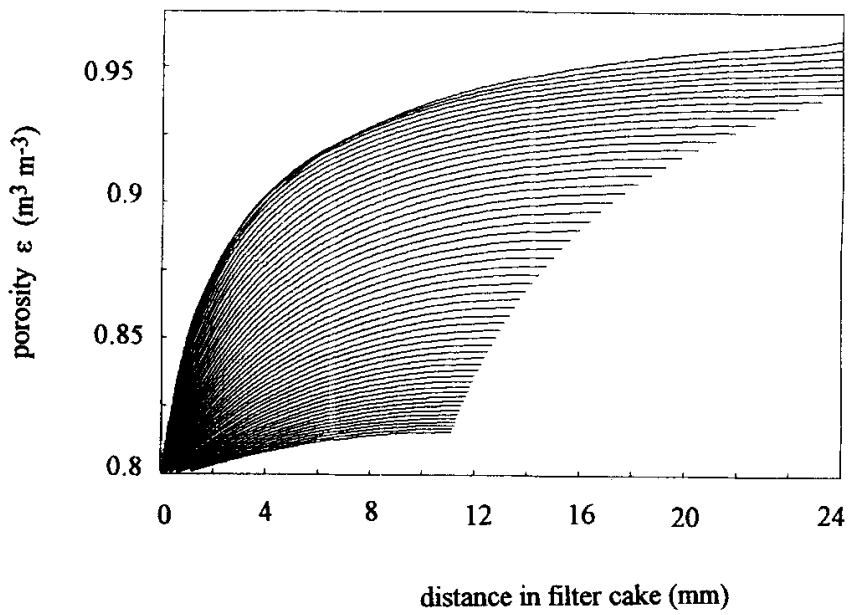

Figure 6. Numerically calculated porosity profiles as a function of time for the expression phase.

Boundary condition $p=100 \mathrm{kPa}$; time between the subsequent profiles is $160 \mathrm{~s} K_{0}=3.0 \times 10^{-13} \mathrm{~m}^{2}, \epsilon_{0}=0.97, p_{a}=$ $1,000 \mathrm{~Pa}, \delta=1.8, \lambda=0.15$.

porosity profiles (Figures 5 and 6, respectively) reveals several interesting features:

(1) According to the experiments, the porosity near the filter medium $(x=0)$ does not rapidly decrease to the equilibrium porosity $\epsilon_{\infty}$, but varies during both the filtration and the expression phase. The resistance of the filter medium $\left(R_{m}\right)$ is thus higher than expected, which may indicate that clogging occurs. By only measuring the filtrate volume, this effect will not be detected.

(2) According to the calculations, subsequent profiles are almost equidistant at the beginning of the expression phase. According to the experiments a retardation occurs: the profiles hardly change during certain time intervals. This is more clearly seen in Figure 7, where the porosity vs. time is plotted for two positions in the cake. After a certain time $( \pm 3,200 \mathrm{~s}$ in this case), the rate at which the porosity decreases suddenly increases again. This phenomenon was observed both

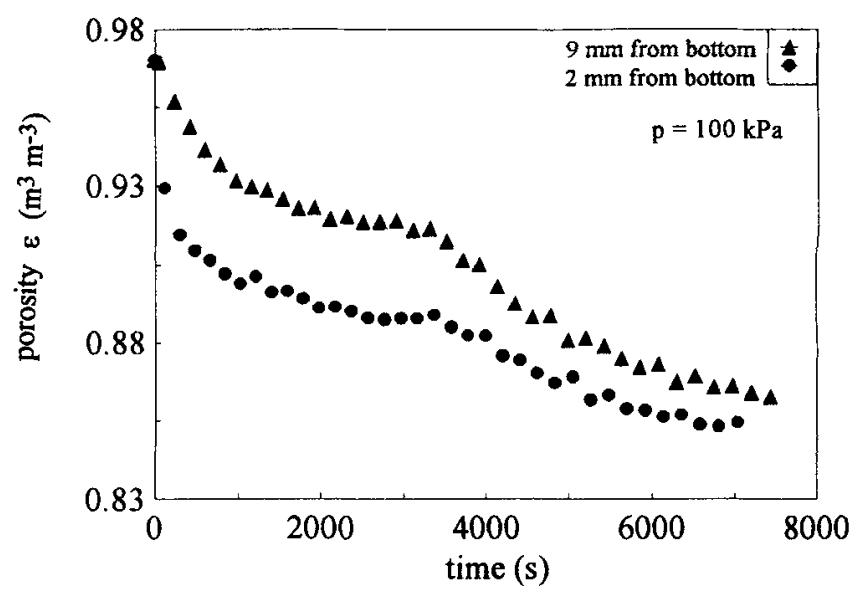

Figure 7. Decrease of the local porosity at two positions in the filter cake ( $2 \mathrm{~mm}$ (dots) and $9 \mathrm{~mm}$ (triangles), measured the from filter medium) plotted against the time. 
with and without a piston, which means that this retardation is not caused by friction between the piston and the wall. This retardation was not observed by only measuring the average decrease of the porosity (determined from the average mass of the cake) and cannot be described by the model discussed above.

(3) There is no sharp transition (discontinuity) visible between the porosity of the slurry and that of the filter cake, as is theoretically assumed (see Figure 2, difference $\epsilon_{s l}-\epsilon_{0}$ ). This indicates that the difference between the porosity of the slurry $\left(\epsilon_{s l}\right)$ and the porosity at the top of the filter cake $\left(\epsilon_{0}\right)$ must be very small. Although the value of the porosity of the slurry can be calculated, the unstressed porosity cannot exactly be determined, as discussed above. The NMR apparatus could be an interesting apparatus to determine this value. Although it is not possible to carry out an NMR experiment with an absolute accuracy better than $1 \%$, smaller changes and certainly a step in porosity can be detected, since the calibration curve is monotonically rising (Figure 4). However, no discontinuity was visible; therefore, the difference between the values of $\epsilon_{s l}$ and $\epsilon_{0}$ must be smaller than 0.01 , assuming that the theoretical concept of a discontinuity is right. This small difference was also confirmed by measurements carried out with the compression-permeability cell ( $\mathrm{La}$ Heij, 1994).

In Figures 8 and 9 experimentally determined porosity profiles are shown as a function of time for two different applied pressures. In this case the sludge was flocculated with 7 wt. $\%$ iron chloride and $50 \mathrm{wt} . \%$ lime, which results in a higher permeability and a lower compressibility (lower values for $\delta$ and $\lambda$ ). The time between the subsequent profiles is again $160 \mathrm{~s}$. The behavior of the porosity profiles as a function of time is almost exactly the same for the two different pressures. Only the final equilibrium porosity $\epsilon_{\infty}$ (see Figure 2) is lower and thus the final cake thickness is smaller for a higher applied pressure. This is in agreement with the filtration-expressed model. It is also clear that, compared to the experimental profiles shown in Figure 5, not only is the total dewa-



Figure 8. Porosity profiles in the filter cake as determined for sludge flocculated with $7 \mathrm{wt}$. \% iron chloride and $\mathbf{5 0}$ wt. \% lime on dry solids base.

The time between the subsequent profiles is $160 \mathrm{~s}$; total time of experiment is $56 \mathrm{~min}$. Boundary condition (applied pressure) $p=50 \mathrm{kPa}, \omega=1.10^{3} \mathrm{~Pa} \cdot \mathrm{s}, \epsilon_{s l}=0.97$ (with flocculant), $\epsilon_{s l}=0.98$ (without flocculant).

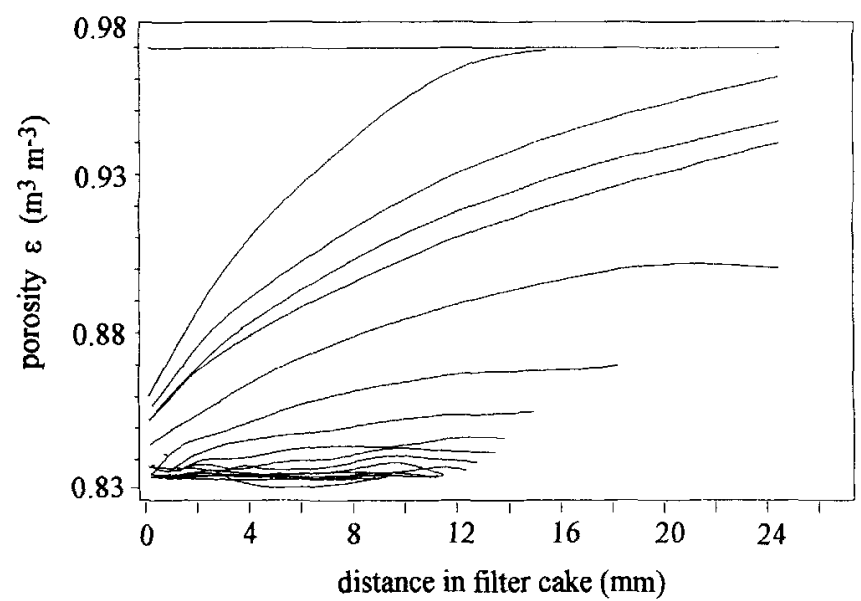

Figure 9. Porosity profiles in the filter cake as determined for sludge flocculated with $7 \mathrm{wt}$. \% iron chloride and $\mathbf{5 0}$ wt. \% lime on dry solids base.

The time between the subsequent profiles is $160 \mathrm{~s}$. Total time of experiment is $40 \mathrm{~min}$. Boundary condition (applied pressure) $p=100 \mathrm{kPa}, \omega=1.10^{3} \mathrm{~Pa} \cdot \mathrm{s}, \epsilon_{s l}=0.97$ (with flocculant), $\epsilon_{s l}=0.98$ (without flocculant).

tering time shorter (higher permeability), but also the slopes of the porosity profiles are less steep. This is caused by the lower compressibility, which reveals that effects of compressibility can be determined by use of NMR imaging as well.

As stated above, the filter medium resistance $R_{m}$ is higher than expected, which may indicate clogging. However, the observed porosity decreases to the equilibrium porosity $\epsilon_{\infty}$ in contrast to the results of model calculations in which an increase of the filter medium resistance with time (clogging) is included. These calculations predict a liquid pressure drop over the filter medium $\Delta p_{l m}$, and a porosity near the filter medium that is higher than the equilibrium porosity $\epsilon_{\infty}$. Obviously, the decrease of the porosity near the filter medium during the expression phase must be determined experimentally.

We like to note that the qualitative deviations between the calculated and the measured profiles (Figures 5 and 6) can also be caused by the fact that the concept of compressibility coefficients (Eqs. 8 and 9), which determine the shape of the calculated porosity profiles, is too simple. This indicates that it is probably better to determine the constitutive equations from the experimental porosity profiles. However, more research is needed to confirm this hypothesis.

\section{Conclusions}

Using NMR scanning, quantitative porosity profiles were measured for the first time during filtration and expression of solid-liquid mixtures. The potential applications of this technique are very diverse, such as checking the constitutive parameters or boundary conditions. Calibration measurements on samples with uniform porosity profiles revealed that for an echo time of $1,800 \mu \mathrm{s}$ a good porosity contrast could be obtained. The nonlinear relation between NMR intensity and porosity is related to the increasing fraction of free water at higher porosities.

From the measured profiles during filtration and expression, it can be concluded that the resistance of the filter medium is higher than theoretically expected. Probably some 
clogging occurs and the porosity near the filter medium varies during both the filtration and expression phase. To determine the constitutive equations, measurements and calculations of porosity profiles of materials with different compression characteristics form an interesting extension of this work.

\section{Acknowledgment}

This work is financially supported by the Institute of Inland Water Management and Waste Water Treatment (RIZA) and the Foundation for Applied Waste Water Research (STOWA) in The Netherlands.

\section{Notation}

$K=$ permeability, $\mathrm{m}^{2}$

$K_{m}=$ permeability of filter medium, $\mathrm{m}^{2}$

$K_{0}=$ permeability at $p_{s}=0, \mathrm{~m}^{2}$

$K_{\infty}=$ equilibrium permeability, $\mathrm{m}^{2}$

$p=$ applied filtration-expression pressure, $\mathrm{Pa}$

$p_{a}=$ reference pressure, $\mathrm{Pa}$

$\Delta p_{l m}=$ hydraulic pressure drop over filter medium, $\mathrm{Pa}$

$p_{s}=$ compressive pressure, $\mathrm{Pa}$

$p_{s . x=0}=$ compressive pressure near filter medium, $\mathrm{Pa}$

$q_{l}=$ superficial liquid velocity, $\mathrm{m} \cdot \mathrm{s}^{-1}$

$\mathrm{RF}=$ radio frequency, $\mathrm{Hz}$

$R_{m}=$ resistance filter medium, $\mathrm{m}^{-1}$

$S=$ NMR signal

$T_{1}=$ longitudinal relaxation time, $\mathrm{s}$

$T_{2}=$ transverse relaxation time, $\mathrm{s}$

$T_{E}=$ echo time, $\mathrm{s}$

$T_{R}=$ repetition time, $\mathrm{s}$

$\Delta x_{m}=$ thickness of filter medium, $\mathrm{m}$

$v_{i}=$ averaged liquid pore velocity, $\mathrm{m} \cdot \mathrm{s}^{-1}$

$v_{\mathrm{s}}=$ averaged solids pore velocity, $\mathrm{m} \cdot \mathrm{s}^{-1}$

\section{Greek letters}

$\delta=$ compressibility coefficient

$\epsilon=$ porosity, $\mathrm{m}^{3} \mathrm{~m}^{-3}$

$\epsilon_{0}=$ porosity at $p_{s}=0, \mathrm{~m}^{3} \mathrm{~m}^{-3}$

$\epsilon_{\infty}=$ equilibrium porosity, $\mathrm{m}^{3} \mathrm{~m}^{-3}$

$\eta=$ viscosity liquid, $\mathrm{Pa} \cdot \mathrm{s}$

$\lambda=$ compressibility coefficient

$\rho=$ proton density, $\mathrm{m}^{-3}$

$\omega=$ solids volume in cake per unit cross sectional area, $\mathrm{m}$

\section{Literature Cited}

Bierck, B. R., S. A. Welis, and R. I. Dick, "Compressible Cake Filtration: Monitoring Cake Formation and Shrinkage Using Synchrotron X-Rays," J. Water Pollut. Control Fed., 60, 645 (1988).

Chase, G. G., and M. S. Willis, "Flow Resistance in Filter Cake Due to Air," Sep. Sci. Technol., 26, 117 (1991).

Hayashi, K., K. Kawashima, K. Kose, and T. Inouye, "NMR Imaging of Advanced Ceramics During the Slip Casting Process," J. Phys. D: Appl. Phys., 21, 1037 (1988).

Horsfield, M. A., E. J. Fordham, and L. D. Hall, "H NMR Imaging Studies of Filtration in Colloidal Suspensions," J. Magnetic Resonance, 81, 593 (1989).

Kopinga, K., and L. Pel, "One Dimensional Scanning of Moisture in Porous Materials with NMR," Rev. of Sci. Instr., 65, 3673 (1994).

La Heij, E. J., "An Analysis of Sludge Filtration and Expression," PhD Thesis, Eindhoven Univ. of Technology, The Netherlands (1994).

La Heij, E. J., P. J. A. M. Kerkhof, A. J. M. Herwijn, and W. J. Coumans, "Fundamental Aspects of Sludge Filtration and Expression," Water Res., accepted (1995).

Rietema, K., "Study on Compressibility of Filter Cakes," PhD Thesis, Delft Univ. of Technology, The Netherlands (1953).

Shirato, M., T. Aragaki, R. Mori, and K. Sawamoto, "Predictions of Constant Pressure and Constant Rate Filtrations Based Upon an Approximate Correction for Side Wall Friction in Compression Permeability Cell Data," J. Chem. Eng. Japan, 1, 86 (1968).
Shirato, M., and T. Aragaki, "Verification of Internal Flow Mechanism Theory of Cake Filtration," Filtration and Sep., 9, 294 (1972).

Stamatakis, K., and C. Tien, "Cake Formation and Growth in Cake Filtration," Chem. Eng. Sci., 46, 1917 (1991).

Tiller, F. M., J. R. Crump, and F. Ville, "A Revised Approach to the Theory of Cake Filtration," Proc. Int. Symp., 2, 1549, P. Somassundarem, ed., AIME (1980).

Tiller, F. M., and C. S. Yeh, "The Role of Porosity in Filtration. Part XI: Filtration Followed by Expression," AIChE J., 33, 1241 (1987). Tiller, F. M., N. B. Hsyung, Y. L. Shen, and W. Chen, "Catscan Analysis of Sedimentation and Constant Pressure Filtration," Proc. World Filtration Congress, Nice, France (1990).

Wakeman, R. J., "The Formation and Properties of Apparently Incompressible Filter Cakes under Vacuum on Downward Facing Surfaces," Trans. Inst. Chem. Eng., 59, 260 (1981).

\section{Appendix: Derivation of Eq. 1}

The partial differential equation describing the change of the porosity in time and place is derived from three basic equations: the Darcy equation, a mass balance, and a force balance. The Darcy equation is

$$
v_{l}-v_{s}=\frac{1}{\epsilon} \frac{K}{\eta}\left(\frac{\partial p_{l}}{\partial x}+\rho_{l} g\right)
$$

where $v_{l}$ and $v_{s}$ are the local averaged pore velocities of respectively the liquid and the solids. A mass balance between an arbitrary point at distance $x$ and $x+d x$ in the filter cake leads to the following continuity equation for the liquid phase

$$
\left(\frac{\partial q_{l}}{\partial x}\right)_{t}=\left(\frac{\partial \epsilon}{\partial t}\right)_{x}
$$

where $q_{l}$ is the superficial liquid velocity. A simple force balance leads to the relation between the hydraulic pressure $p_{l}$ and the compressive pressure $p_{s}$

$$
\frac{\partial p_{l}}{\partial x}+\frac{\partial p_{s}}{\partial x}+\left(\rho_{l} \epsilon+\rho_{s} \epsilon_{s}\right) g=0
$$

Combination of Eqs. A1, A2, and A3 leads to the partial differential equation describing the change of the porosity in time and place in a filter cake

$$
\left(\frac{\partial \epsilon}{\partial t}\right)_{x}=q_{l m}\left(\frac{\partial \epsilon}{\partial x}\right)_{t}+\frac{\partial}{\partial x}\left\{\frac{K}{\eta}(1-\epsilon)\left[\left(\rho_{s}-\rho_{l}\right) g-\left(\frac{\partial p_{s}}{\partial x}\right)\right]\right\}
$$

Since during filtration a filter cake builds up, dimensionless coordinates $[x / L(t)]$ can be used and a moving boundary (Stamatakis and Tien, 1991) has to be applied to solve Eq. A4. During expression, the amount of solids remains constant and therefore solid-based coordinates can be used ( $\mathrm{La}$ Heij, 1994)

$$
d \omega=(1-\epsilon) d x
$$

By introducing Eq. A5 and neglecting gravity effects (which is debatable at low applied pressures), Eq. A4 will change into Eq. 1.

Manuscript received Jan. 6, 1995, and revision received July 18, 1995 